\title{
Campylobacter pylori infection in biopsy specimens of gastric antrum: laboratory diagnosis and estimation of sampling error
}

\author{
A MORRIS, * M R ALI, $\dagger$ PAMELA BROWN, $\dagger$ MLANE, $\dagger$ KATHY PATTON \\ From the Departments of ${ }^{*}$ Microbiology and $\dagger$ Gastroenterology, Middlemore Hospital, and the $\ddagger$ Department of \\ Pathology, Auckland Hospital, Auckland, New Zealand
}

SUMMARY Campylobacter pylori infection was sought in 382 consecutive patients referred for upper gastrointestinal endoscopy. Five antral biopsy specimens were taken from each patient: one was inserted into a CLO-test to detect the urease activity of $C$ pylori, two were sent for histological analysis where multiple sections were stained by the Warthin-Starry silver method, and two were sent for microbiological evaluation by Gram stain and culture. A patient was deemed to be infected when $C$ pylori was cultured or seen in either the histological sections or the Gram stain of the biopsy smear. One hundred and seventy four (46\%) patients were infected. Culture, Gram stain, histological examination and the CLO-test showed sensitivities of $92 \%, 87 \%, 93 \%$ and $90 \%$, respectively. In 27 $(15 \%)$ infected patients an uneven distribution of $C$ pylori was seen between samples in the biopsy pair sent for histology. Examination of multiple sections stained with Warthin-Starry silver was more sensitive at detecting infection (93\%) than examination of multiple sections from only one biopsy specimen (84\%). Fifty seven of 80 patients, biopsied a median seven days (range 5 to 55 ) after completing colloidal bismuth subcitrate treatment, were still infected with $C$ pylori. There was no decrease in the sensitivities of the above tests to detect infection after treatment. It is concluded that at least two antral biopsy specimens should be examined when attempting to diagnose $C$ pylori infection by histological methods.

In 1983 an Australian pathologist, Dr J Warren, reported an association between colonisation of the stomach with curved bacilli and the presence of active chronic gastritis. ' Since then a great deal of interest has been shown in this organism, currently known as Campylobacter pylori. ${ }^{2}$ Consequently, many histopathologists and microbiologists are being asked to detect infection with this organism, but there is no commonly acknowledged "gold standard" method for diagnosing $C$ pylori infection.

The quickest way to detect $C$ pylori in histological sections would be to examine one suitably stained section of a biopsy specimen under high power. We undertook a detailed examination of Warthin-Starry silver stained sections to see how sensitive this approach is in detecting $C$ pylori compared with examination of multiple sections from two antral biopsy specimens. The sensitivities of three other tests-culture, Gram stain of biopsy homogenate, and the CLO-test were also calculated. We also looked to see if the sensitivities of the above tests were affected as a result of colloidal bismuth subcitrate treatment.

\section{Material and methods}

Three hundred and eighty two patients referred for routine gastrointestinal endoscopy were biopsied to detect $C$ pylori. Patients were biopsied according to a protocol approved by the Middlemore Hospital ethical committee. The association between infection and histological gastritis and endoscopic appearances of the stomach are reported elsewhere. ${ }^{3}$ This report details only the data on the diagnostic ability of four tests to detect $C$ pylori infection. Eighty patients entering treatment trials using colloidal bismuth subcitrate (CBS) were biopsied after completing four weeks of treatment. Fifty seven patients were still infected after treatment and the results of these patients' biopsy specimens were recorded. Five antral biopsy specimens taken close together were obtained from each patient within $5 \mathrm{~cm}$ of the pylorus. 
HISTOLOGICAL ANALYSIS

Two antral biopsy specimens from each patient were fixed in $10 \%$ buffered formalin. Paraffin wax sections $4 \mu \mathrm{m}$ thick were cut and stained by the Warthin-Starry silver method. ${ }^{4}$ Staining was performed in a darkened room to avoid excessive silver precipitation. When a batch of sections was stained too lightly or if interpretation was hindered by excessive silver precipitate, extra sections were cut and stained. The entire epithelial surface of all stained sections was examined under oil immersion by one observer who did not know the results of the patient's other tests. The presence of curved bacilli in the vicinity of the gastric epithelium was taken to indicate $C$ pylori infection. The presence or absence of organisms in each section of each biopsy specimen was recorded.

\section{MICROBIOLOGY}

Two antral biopsy specimens were placed in $12 \mathrm{ml}$ of $12.5 \%$ dextrose and transported to the laboratory for processing within three hours of collection. The methods used were those previously published. ${ }^{356}$ In short, the specimens were placed in a drop of transport solution on a clean glass slide and finely minced with sterile scalpel blades. Firm pressure was used to form a smear of the homogenate. Most of the homogenate was then removed with a cotton tipped swab and smeared on to the surface of a modified Skirrow's Camplyobacter media (Columbia base) containing polymixin B (5 IU $/ \mathrm{ml})$, trimethoprim $(10 \mu \mathrm{g} / \mathrm{ml})$, and vancomycin $(10 \mu \mathrm{g} / \mathrm{ml})$. Plates were made every 10 to 14 days and stored at $4^{\circ} \mathrm{C}$ in sealed plastic bags before use. Plates were incubated in anaerobic jars which were evacuated to $520 \mathrm{mmHg}$ and flushed with a gas mixture of $10 \%$ carbon dioxide and $90 \%$ nitrogen. Plates were kept for at least five days before being discarded. $C$ pylori was identified by colonial morphology, and by positive catalase, oxidase, and urease reactions. The material remaining on the glass slide was air-dried, heat fixed, and Gram stained.

\section{UREASE DETECTION}

One biopsy specimen was placed into the urea containing agar of the CLO-test (Delta-West, Western Australia). ${ }^{78} \mathrm{CLO}$-tests changing from yellow to orange or deep pink within 24 hours were read as positive. A patient was defined as being infected if $C$ pylori was cultured or if characteristically shaped organisms were seen in the Gram stain of the biopsy smear or in the sections stained with Warthin-Starry silver. We took this definition as our "gold standard". A positive CLO-test by itself was not taken as definite evidence of infection.

Comparison between tests was by two-by-two contingency tables using the $\chi^{2}$ test. $P$ values of $<0.05$ were considered to be significant.

\section{Results}

Of the 382 patients biopsied, $174(46 \%)$ were infected. Table 1 shows the test results for all patients. The sensitivity of the tests in dectecting infection in the 174 routine patients is shown in table 2 . Combined microbiological results - that is, Gram stain of the biopsy smear and culture-were more sensitive at diagnosing infection (97\%) compared with the CLOtest $(90 \%)(p<0.02)$, or Gram stain alone $(87 \%)$ $(p<0.002)$. Test results for patients biopsied after treatment with colloidal bismuth subcitrate are also shown in table 2 . Fifty seven of 80 patients $(71 \%)$ were still infected when biopsied a median seven days (range five to 55 days) after completing a four week course of treatment (Morris, Ali, Brown, unpublished observations). ${ }^{3}$ Most patients, 64 of 80 (80\%), were biopsied seven to 10 days after treatment. There was no decreased sensitivity in any test for diagnosing infection in antral biopsy specimens taken a median of seven days after treatment.

All four tests were positive in three quarters of infected patients; only $5 \%$ of infected patients were positive in one test alone (tables 1 and 2). Of the 10 routine patients positive by one test alone, five had active chronic gastritis, four had chronic gastritis, and only one, the patient positive by Gram stain alone, had normal antral histology. Positive CLO-test results were observed in two patients in whom no definitive evidence of $C$ pylori was present (table 1). The tests were positive three and 24 hours after endoscopy. Neither patient had histological gastritis.

Table 1 Diagnostic rest results in 382 patients biopsied to detect Campylobacter pylori infection*

\begin{tabular}{llllll}
\hline \multicolumn{2}{l}{ Diagnostic test $\dagger$} \\
\hline Culture & Gram stain & Silver stain & CLO-test $\neq$ & Number \\
\hline+ & + & + & + & 135 \\
+ & + & + & - & 2 \\
+ & - & + & + & 8 \\
- & + & + & + & 5 \\
+ & + & - & + & 6 \\
- & - & + & + & 2 \\
+ & - & + & - & 3 \\
+ & + & - & - & 1 \\
- & + & + & - & 2 \\
- & + & - & - & 1 \\
+ & - & - & - & 5 \\
- & - & + & - & 206 \\
- & - & - & + & 2 \\
- & - & - & & 382 \\
& & & & & - \\
\hline
\end{tabular}

*Infection defined by positive culture, or seeing the organism in either the Gram stain smear or silver stained histological sections. $+t=$ positive test, $-=$ negative test. $160(92 \%), 152(87 \%), 161$ $(93 \%)$ and $156(90 \%)$ infected patients were positive by culture, Gram stain, silver stain and CLO-test, respectively. †LLO-test result 24 hours after endoscopy. 
Table 2 Percentage sensitivity of diagnostic tests in detecting Campylobacter pylori infection in routine gastroscopy patients and in patients completing treatment with colloidal bismuth

\begin{tabular}{lcc}
\hline & \multicolumn{2}{l}{ Patients } \\
\cline { 2 - 3 } Diagnostic test & Routine* & Treated \\
\hline & $(\mathrm{n}=174)$ & $(\mathrm{n}=57)$ \\
Culture & 92 & 96 \\
Gram stain & 87 & 86 \\
Gram stain and/or culture & 97 & 100 \\
Silver stain & 93 & 88 \\
CLO-test & 90 & 88 \\
All four tests positive & 78 & 74 \\
Three or more tests positive & 89 & 88 \\
Two or more tests positive & 94 & 96 \\
One or more tests positive & 100 & 100 \\
Only culture positive $\ddagger$ & 5 & 2 \\
Only Gram stain positive $\ddagger$ & 1 & 0 \\
Only Warthin-Starry stain positive $\ddagger$ & 4 & 0 \\
\hline
\end{tabular}

*One hundred and seventy four patients of 382 consecutive patients presenting for endoscopy were infected with $C$ pylori. Infection defined by positive culture or seeing the organism in Gram stain smear or silver stained histological sections.

tTreated patients had completed a four week course of colloidal bismuth subcitrate. Patients were biopsied, a median of seven days (range six to 55 days) after treatment: 44 of $57(77 \%)$ were biopsied seven to 10 days after treatment.

$\ddagger$ Number of patients.

Each patient had a median of four (range two to 10) Warthin-Starry silver sections from each biopsy specimen examined under oil immersion: four patients were positive by this method alone (tables 1 and 2). One hundred and thirty four of the $174(77 \%)$ infected patients had organisms present in all sections of both biopsy specimens. In 13 infected patients (7\%) neither biopsy specimens contained organisms. Twenty seven $(15 \%)$ infected patients had uneven distribution of $C$ pylori. In $16(9 \%)$ all sections in one biopsy specimen but no section in the other biopsy specimen contained organisms. In $11(6 \%)$ patients more variable distribution of $C$ pylori was seen (table 3 ). In five patients each biopsy specimen contained organisms but not every section in each biopsy specimen. In three patients each section in one biopsy specimen contained organisms but not all sections in the other biopsy specimen; in three other patients no organisms were seen in one biopsy specimen, and while the other biopsy specimen contained $C$ pylori, not every section contained organisms. The variation between adjacent sections was associated with low numbers of organisms being present in positive sections. Such numbers would have been missed with a lower power objective, or if the entire epithelial surface had not been examined. In these 11 patients with variable distribution of $C$ pylori 42 of the 92 sections (46\%) examined contained organisms - that is, about half of the biopsy specimens in this group were positive.

Estimation of the sensitivity of examining one or multiple sections from one or two biopsy specimens
Table 3 Distribution of Campylobacter pylori in 11 patients with variable distribution or organisms within antral biopsy specimens*

\begin{tabular}{lll}
\hline $\begin{array}{l}\text { Both biopsy } \\
\text { specimens with } \\
\text { variable distribution }\end{array}$ & $\begin{array}{l}\text { One biopsy } \\
\text { specimen positive, } \\
\text { other variable }\end{array}$ & $\begin{array}{l}\text { One biopsy } \\
\text { specimen negative, } \\
\text { other variable }\end{array}$ \\
\hline+----+ & +++++ & --- \\
--++++ & +---- & -++ \\
+--- & ++++ & ---- \\
---+ & ++-- & -++- \\
-+++ & +++++ & ------ \\
---+ & +++-- & +----+ \\
+-- & & \\
++- & & \\
++--+ & & \\
\hline
\end{tabular}

*Number of sections examined in each patient represented by the number of + and - symbols: $+=$ organisms seen in the section, $-=$ no organisms seen in the section.

†Tissue of only one biopsy specimen remained in the block for sectioning and staining.

was made by assuming that there was an even chance of obtaining either biopsy specimen if only one had been taken. If only one stained section from one biopsy specimen had been examined 147 of the 174 $(84 \%)$ infected patients would have been detected (134 with organisms in each section of both biopsy specimens, eight of the 16 with only one biopsy specimen positive, and five of the 11 patients with variable distribution of organisms). The same number would have been detected if multiple sections from only one biopsy specimen had been examined. If only one stained section had been examined from both biopsy specimens $157(90 \%)$ infected patients would have been detected (134 with organisms in each section, all 16 with one positive biopsy specimen and seven of the patients with variable distribution of $C$ pylori: all three with all sections in one biopsy specimen were positive and four of the remaining eight with variable distribution).

Examination of multiple sections from both biopsy specimens detected infection in 161 of the $174(93 \%)$ infected patients. Comparison of the above approaches showed that examination of multiple sections from two biopsy specimens had a greater sensitivity $(93 \%)$ than examining either one or multiple sections from only one biopsy specimen $(84 \%)(p<0.03)$. The sensitivity of examining one section from both biopsy specimens $(90 \%)$ was not more significant than examining one biopsy specimen alone $(84 \%)$, $\mathrm{p}=0 \cdot 21$.

Review of published data identified 13 papers $^{8-20}$ which contained enough detail to determine how often $C$ pylori was detected in one biopsy specimen when another specimen taken at the same time was negative (table 4). This finding ranged from $0 \%-43 \%$, median $14 \%$. 
Table 4 Review of published data on laboratory diagnosis of Campylobacter pylori infection*

\begin{tabular}{|c|c|c|c|c|c|}
\hline \multirow[b]{2}{*}{ Reference } & \multirow{2}{*}{$\begin{array}{l}\text { Patients } \\
\text { infected/total }\end{array}$} & \multirow{2}{*}{$\begin{array}{l}\text { Percentage } \\
\text { infected }\end{array}$} & \multicolumn{2}{|c|}{ No of biopsy specimens } & \multirow[b]{2}{*}{ Comment } \\
\hline & & & Histology & Culture & \\
\hline $\begin{array}{l}\text { Marshall et al } \\
\text { Jones et al } \\
\text { Langenburg et al } \\
\text { McNulty, Wilson } \\
\text { Goodwin et al }{ }^{13}\end{array}$ & $\begin{array}{l}56 / 96 \dagger \\
31 / 50 \\
32 / 50 \\
47 / 83 \\
60 / 1035\end{array}$ & $\begin{array}{l}58 \\
62 \\
64 \\
57 \\
58\end{array}$ & $\begin{array}{l}1 \\
\text { NS } \\
\text { NS } \\
\text { NS } \\
1\end{array}$ & $\begin{array}{l}1 \\
\text { NS } \\
\text { NS } \\
\text { NS } \\
1\end{array}$ & \multirow{2}{*}{$\begin{array}{l}24(43 \%) \text { positive in one biopsy specimen alone } \\
11(35 \%) \text { positive by only histology or culture } \\
1(3 \%) \text { positive by culture alone } \\
4(9 \%) \text { positive by histology alone } \\
11(18 \%) \text { positive in only one of the biopsy } \\
\text { specimen pair } \\
5(16 \%) \text { positive in one biopsy specimen alone } \\
\text { No patient positive in one biopsy specimen alone } \\
5(10 \%) \text { positive by microbiology alone } \\
14(6 \%) \text { positive by histology alone } \\
4(19 \%) \text { positive in one biopsy specimen alone } \\
7(9 \%) \text { positive by histology alone } \\
9(16 \%) \text { positive by one biopsy specimen alone } \\
10(14 \%) \text { positive by only histology or culture } \\
27(15 \%) \text { uneven distribution between biopsy } \\
\text { specimens of the same pair }\end{array}$} \\
\hline 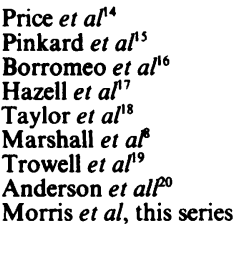 & $\begin{array}{c}32 / 51 \\
48 / 80 \\
51 / 80 \\
218 / 376 \\
21 / 57 \\
79 / 141 \\
57 / 102 \\
71 / 153 \\
174 / 382\end{array}$ & $\begin{array}{l}63 \\
80 \\
64 \\
58 \\
41 \\
56 \\
56 \\
46 \\
46\end{array}$ & $\begin{array}{l}1 \\
1 \\
1 \ddagger \\
1 \\
1 \\
2 \\
1 \\
\text { NS } \\
2\end{array}$ & $\begin{array}{l}1 \\
2 \\
2 \\
1 \ddagger \\
1 \\
1 \\
1 \\
\text { NS } \\
2\end{array}$ & \\
\hline
\end{tabular}

* Only papers with sufficient detail in the material and methods or result sections are included.

fOnly the data pertaining to the 96 patients with paired biopsy specimens sent for histological assessment and Gram staining are shown.

$\$ 103$ biopsy specimen pairs obtained from 80 patients.

¡Biopsy used for CLO-test.

NS = Number of biopsy specimens not stated.

\section{Discussion}

Many different protocols have been used to search for $C$ pylori in biopsy specimens. ${ }^{368-20}$ Usually one specimen is sent for histological assessment, ${ }^{93-161718}$ but sometimes two are sent. ${ }^{358}$ Although routine processing of biopsy specimens for haematoxylin and among eosin staining could be expected to be reasonably comparable laboratories, many other different stains are used to detect $C$ pylori in tissue sections. ${ }^{51921}$ The Warthin-Starry silver method is time consuming, expensive, and needs experience to obtain optimal results. Some batches of slides have to be repeated because of pale staining and others because excessive precipitate prevents adequate inspection of the epithelial surface. We have found the half $\mathrm{Gram}^{19}$ and Giemsa $^{21}$ methods are as sensitive as the WarthinStarry method and far easier to perform (Morris, Patton, unpublished observations). We suspect that most reported methods, once shown to work with adequate control material, perform equally well in their ability to detect $C$ pylori. Apart from the staining methods it is difficult to comment on how reliable histological assessment has fared in detecting $C$ pylori because the manner of inspection - that is, high dry or oil immersion, or more importantly, the number of sections inspected for each biopsy specimen-have not been detailed in published reports.

Far greater variation exists in the methods used to detect $C$ pylori by Gram stain or culture. ${ }^{38-20}$ Variations exist in the number of biopsy specimens submitted, transport media used, preparation of Gram stain or culture inoculum (such as smearing or rubbing the biopsy specimen over a slide or agar plate rather than dispersing the tissue by cutting or grinding), and type of media (selective or non-selective), number of antibiotics in selective media and atmospheric conditions (gas generating systems or flushing evacuated anaerobic jars with gas mixtures). Many methods have also been successful in detecting the urease activity of Cpylori. ${ }^{78172223}$ We used the CLO-test because we had previously found it to be reliable, ${ }^{7}$ but other methods are cheaper, yet share the CLO-test's reliability and speed. ${ }^{1722} 23$

In this study we found that microbiological methods identified most infected patients $(\mathbf{9 7 \%})$ presenting for routine gastroscopy (table 2). Three quarters of infected patients were positive in all four tests and only $5 \%$ were positive by one test alone. It has been reported that false positive culture results can occur due to contamination of biopsy material via the endoscope or biopsy forceps when these have been used on an infected patient immediately before. ${ }^{24}$ The finding of histological gastritis in nine of the 10 patients positive by only one test suggests that contamination was not the reason for isolated positive results in our patients.

Previous reports have not detailed the way in which $C$ pylori was sought in histological sections and in particular have not reported the number of sections examined from each biopsy specimen. We have found that examination of multiple sections (mean four) from two antral biopsy specimens detected infection in $93 \%$ of infected patients. Examination of multiple sections from two biopsy specimens was more sensitive at detecting infection $(93 \%)$ than examination of one alone $(84 \%)(p<0.03)$. Moreover, we found that $9 \%$ of infected patients have organisms present in one biopsy specimen but not the other taken at the same 
time. A further $6 \%$ of infected patients have even more variable distribution of organisms where sequential sections within the same biopsy specimen may show varying results (table 3 ). We feel that our findings on the uneven distribution of $C$ pylori are due to variations in bacterial numbers within antral biopsy specimens and do not represent any particular weakness or strength of the Warthin-Starry silver method itself.

These results prompted a review of published data to see if there was any other evidence for the frequently made claim that the distribution of $C$ pylori may be uneven or patchy. ${ }^{11317182025}$ The percentage of patients positive in one biopsy specimen but not in another taken at the same time is about $14 \%$ (table 4). Several authors give additional details of uneven distribution of $C$ pylori. ${ }^{131725}$ Goodwin et al observed that most histological sections showed an uneven distribution of bacteria, with the distribution often being quite patchy. ${ }^{13}$ In 21 infected patients from whom two or more biopsy specimens had been examined histologically, $13(62 \%)$ showed different numbers of bacteria among specimens, and in two cases $(10 \%)$ bacteria were not seen in one or more biopsy specimens. ${ }^{13}$ In the large series reported by Hazell et al one antral biopsy specimen was routinely submitted for histological assessment, but in one case where two antral biopsy specimens were sent, bacteria were seen in one biopsy specimen but not in the other. ${ }^{17}$ Karttumen et al observed two cases where sections stained with haematoxylin and eosin were negative and sections stained with Warthin-Starry were positive, but not all the latter sections contained organisms. ${ }^{25}$

The biopsy test results from patients who had been treated with colloidal bismuth are interesting. These patients had completed four weeks of colloidal bismuth treatment (Morris, Ali, Brown, unpublished observations). ${ }^{3}$ There was no apparent reduction in the sensitivity of these tests in diagnosing infections in antral biopsy specimens obtained a median of seven days after stopping treatment. Yet relapse of infection was observed in patients in these treatment trials whose initial biopsy specimens after treatment were negative. ${ }^{3}$ Either relapse of infection is due to reinfection of the patient from an external source soon after treatment or to recrudescence of infection from within the stomach. If relapse is due to recrudescence of infection from the antrum then this would imply that our biopsy methods missed low grade antral infection. If this is true then it would imply that our biopsy protocol could also miss infections with similar numbers of organisms in other patients. In this series of 382 routine patients there were 27 patients with active chronic gastritis in whom no evidence of $C$ pylori infection was found. ${ }^{3}$ These results suggest that either there are other causes of active chronic gastritis or that five antral biopsy specimens may still miss infection in some patients.

The number of biopsy specimens taken and the diagnostic tests used to detect $C$ pylori will depend on the motivation for the biopsy series and the availability of local diagnostic services. For most patients undergoing upper gastrointestinal endoscopy it has yet to be shown that detection of $C$ pylori is important in patient management. For patients with duodenal ulceration, however, the situation may be different. Recent reports suggest that the persistence of $C$ pylori after treatment is an important predictor of relapse for recently healed duodenal ulceration. ${ }^{2627}$ It is likely, therefore, that a biopsy to detect $C$ pylori will become more common in this patient group, and clinicians and pathologists will have to decide how they wish to diagnose infection. Currently, the simplest and cheapest way is with the use of a reliable urease test. Several methods, entailing only one biopsy specimen being taken, are quick, sensitive, and specific. ${ }^{78172223}$ The cost in terms of time and reagents to increase the detection of $C$ pylori by microbiological or histological techniques are considerable. In our hands, microbiological methods are most sensitive at detecting infection, but they do not provide information on the presence of gastritis. Therefore, some will prefer to use histological methods because they may be used to detect infection as well as allowing grading of gastritis. It is unlikely that all methods will suit the requirements for every situation and in the end the choice may well come down to one of personal preference.

It should be emphasised that the findings presented here refer only to antral biopsy specimens. Other areas of the stomach may have different variation in organism distribution; nevertheless, we have detailed variation in the distribution of $C$ pylori which has important implications for those attempting to detect it. On the basis of our results and the results of our review of published data, we believe that two antral biopsy specimens are better than one for diagnosing $C$ pylori infection. We suggest that if histological methods are used to diagnose infection, multiple sections from each biopsy specimen be examined to ensure optimal detection of $C$ pylori.

Gist-Brocades (NZ) Ltd and Boehringer Ingelheim (NZ) Ltd provided support for the purchase of the CLO-tests.

\section{References}

1 Warren J. Unidentified curved bacilli on gastric epithelium in active chronic gastritis. Lancet 1983;i:1273.

2 Marshall B, Goodwin C. Revised nomenclature of Campylobacter pyloridis. Int J Syst Bacteriol 1987;37:68.

3 Morris A, Brown P, Ali MR, Lane M, Palmer R. Treatment of 
Campylobacter pylori gastritis: a pilot study using pirenzepine dihydrochloride (Gastrozepin) and three formulations of colloidal bismuth substrate (De-Nol). NZ Med J 1988;101: 651-4.

4 Luna L. Manual of histological staining methods of the Armed Forces Institute of Pathology. New York: McGraw-Hill, 1968:238-40.

5 Morris A, Arthur J, Nicholson G. Campylobacter pyloridis infection in Auckland patients with gastritis. NZ Med J 1986;99:353-5.

6 Morris A, Gillies M, Patton K. Detection of Campylobacter pyloridis infection. NZ Med J 1986;99:336.

7 Morris A, McIntyre D, Rose T, Nicholson G. Rapid diagnosis of Cambylobacter pyloridis infection. Lancet 1986;i:149.

8 Marshall B, Warren J, Francis G, Langton S, Goodwin C, Blincow E. Rapid urease test in the management of Campylobacter pyloridis associated gastritis. Am J Gastroenterol 1987;82: 200-10.

9 Marshall B, Warren J. Unidentified curved bacilli in the stomach of patients with gastritis and peptic ulceration. Lancet 1984; i:1311-15.

10 Jones D, Lessells A, Eldridge J. Campylobacter like organisms on the gastric mucosa: culture, histological and serological studies. J Clin Pathol 1984;37:1002-6.

11 Langenburg W, Tytgat G, Schipper M, Rietra P, Zanen H. Campylobacter-like organisms in the stomach of patients and healthy individuals. Lancet 1984;i:1348.

12 McNulty C, Watson D. Special bacteria of the gastric antrum. Lancet 1984;i:1068-9.

13 Goodwin C, Blincow E, Warren J, Waters T, Sanderson C, Easton L. Evaluation of cultural techniques for isolating Campylobacter pyloridis from endoscopic biopsies of gastric mucosa. $J$ Clin Pathol 1985;38:1127-31.

14 Price A, Levi J, Dolby J, Dunscombe P, Smith A, Clark J, Stephenson M. Campylobacter pyloridis in peptic ulcer disease: microbiology, pathology and scanning electron microscopy. Gut 1985;26:1183-8.

15 Pinkard K, Harrison B, Capstick J, Medley G, Lambert J. Detection of Campylobacter pyloridis in gastric mucosa by phase contrast microscopy. J Clin Pathol 1986;39:112-3.

16 Borromeo M, Lambert J, Pinkard K. Evaluation of "CLO-test" to detect Campylobacter pyloridis in gastric mucosa. J Clin Pathol 1987;40:462-3.

17 Hazell S, Borody T, Lee A. Campylobacter pyloridis gastritis I: detection of urease as a marker of bacterial colonisation and gastritis. Am J Gastrolateral 1987;82:292-6.

18 Taylor D, Hargreaves J, Ng L, Sherbaniuk R, Jewell L. Isolation and characterisation of Campylobacter pyloridis from gastric biopsies. Am J Clin Pathol 1987;87:49-54.

19 Trowell J, Yoong A, Saul K, Gant P, Bell G. Simple half-gram stain for showing presence of Campylobacter pyloridis in sections. J Clin Pathol 1987;40:702.

20 Andersen L, Holck S, Povlsen C, Elsborg L, Justesen T. Campylobacter pyloridis in peptic ulcer disease. Scand $J$ Gastroenterol 1987;22:219-24.

21 Gray S, Wyatt J, Rathbone B. Simplified techniques for identifying Campylobacter pyloridis. J Clin Pathol 1986;39:1279-80.

22 McNulty C, Wise R. Rapid diagnosis of Campylobacter pyloridis infection. Lancet 1986;i:387.

23 Arvind A, Cook R, Tabaqchali S, Farthing M. One-minute endoscopy room test for Campylobacter pylori. Lancet 1988;i:704.

24 Lagenberg W, Rauws E, Houthoff $\mathrm{H}$, et al. Follow-up study of individuals with untreated Campylobcter pylori-associated gastritis and of non-infected persons with non-ulcer dyspepsia. $J$ Infect Dis 1988;157:1245-9.

25 Karttunen T, Niemelu S, Lehtola J, Heikkila J, Maentausta O, Rasanen $O$. Campylobacter-like organisms and gastritis: Histology, bile refrux, and gastric fluid composition. Scand J Gastroenterol 1987;22:478-86.

26 Coghlan J, Humphries H, Dooley C, et al. Campylobacter pylori and recurrence of duodenal ulcers-a 12 month follow-up study. Lancet 1987;ii:1109-11.

27 Marshall B, Warren J, Blincow E, et al. Prospective double-blind trial of duodenal ulcer relapse after eradication of Campylobacter pylori. Lancet 1988;ii:1437-42.

Requests for reprints to: Dr A Morris, Microbiology Laboratory, Green Lane Hospital, Green Lane, Auckland, New Zealand. 